\title{
ESTABILIDAD Y DESEMPEÑO DEL COLORANTE ANTOCIANICO EN YOGUR NATURAL
}

STABILITY AND PERFORMANCE OF ANTOCIAN COLORANT IN NATURAL YOGURT

\author{
Andrea Lizeth Lucero Bustos* \\ Angie Pamela Muñoz Betancourt ** \\ FUNDACIÓN UNIVERSIDAD DE AMÉRICA
}

Recibido: 2 de septiembre de $2018 \quad$ Aceptado: 24 de junio de 2019

DOI: https://doi.org/10.29097/23461098.306

\section{Resumen}

Actualmente los colorantes naturales han sido sustituidos por los sintéticos, debido a los beneficios que su consumo genera, además del poder de tinción que proveen a distintos alimentos y que mejoran su apariencia. Por esta razón, este artículo estudia el desempeño de un colorante natural antociánico en un yogur, realizando una comparación con el colorante sintético rojo allura, que es uno de los colorantes más utilizados actualmente en productos alimenticios como jugos y yogures; adicionalmente, se realizaron pruebas de estabilidad al colorante natural para establecer las condiciones adecuadas de almacenamiento. Finalmente, se comprobó que sí hay poder de tinción del colorante en el yogur natural y que en pruebas de estabilidad (de cuatro semanas) permanece la intensidad del color.

Palabras clave: colorante natural, estabilidad, antocianinas, yogur.

\section{Abstract}

Currently, natural dyes have been replaced by synthetics, due to the benefits they may suffer when consumed, in addition to the staining power that different foods have shown improved their appearance. For this reason, this article establishes the performance of a natural anthocyanin dye in a yogurt, making a comparison with the allura red synthetic dye that is one of the most commonly used dyes in different food products such as juices and yogurts, additionally, stability tests to the natural dye will be needed to establish adequate storage conditions. Finally, it is verified that if there is dyeing power of the dye in natural yogurt and in the stability tests for four weeks, the color intensity remains.

Keywords: natural coloring, stability, anthocyanins, yogurt.

\footnotetext{
* Ingeniera química. andrea.lucero@estudiantes.uamerica.edu.co

** Ingeniera química.
} 


\section{INTRODUCCIÓN}

Según Sanz (s. f.), un colorante es un compuesto orgánico:

[...] que al aplicarlo a un sustrato (fibra textil, cuero, papel, plástico o alimento) le confiere un color más o menos permanente, se aplica en disolución o emulsión y el sustrato debe tener cierta afinidad para absorberlo. Los colorantes en general son solubles en el medio en el que se aplican o en el producto final.

Estos compuestos son utilizados en la elaboración de alimentos para ciertos propósitos, a saber:

- Restaurar el color original del alimento, cuando este se haya destruido como efecto de algún proceso tecnológico o condición de almacenaje.

- Corregir variaciones naturales del color, por ejemplo, manteniendo la uniformidad del color debido a variaciones en la intensidad del mismo.

- Intensificar el color propio del alimento cuando este es débil y poco uniforme. Por ejemplo, en yogur adicionados de fruta, el color que esta proporciona es muy débil y resulta poco atractivo a los consumidores.

- Otorgar al alimento un color que lo identifique y le dé una apariencia atractiva. (Parra, 2004, p. 11)

Los colorantes "pueden ser naturales, si son extraídos de una sustancia vegetal, animal o mineral, o sintéticos, si son productos modificados química o físicamente” (Sánchez, 2013, p. 238).

\section{Colorantes artificiales}

Los colorantes artificiales:

[...] requieren de una certificación en la cual se estipulan especificaciones que se deben cumplir para llevar a cabo su implementación. Dichas certificaciones son generadas por diversas autoridades sanitarias y expertos. Antes de aprobar cualquier aditivo, se analizan los datos que existen sobre los efectos en el consumo del colorante, de esta forma es posible definir una ingesta diaria admisible (IDA) con un amplio margen de seguridad. [...] Entre los más utilizados están el amarillo 5 (tartracina), amarillo 6 (amarillo crepúsculo), rojo 2 (amaranto), rojo 40 (rojo allura), y Rojo 3 (eritrosina). (Belmonte, Arroyo, Vázquez, Cruz y Peña, 2016, pp. 25-26)

En cuanto a la seguridad en el uso de los colorantes sintéticos:

[...] se han realizado numerosos estudios que intentan mostrar un panorama general sobre el efecto a la salud que estos producen. A muchos de estos colorantes se les atribuyen perturbaciones en el comportamiento de los niños. Esto no se ha podido comprobar debido a que en muchos casos se consumen mezclas de colorantes, y no se sabe si el efecto es de algún colorante en particular, Sin embargo, tales hechos han generado que su uso en alimentos se limite cada vez más. (Belmonte, Arroyo, Vázquez, Cruz y Peña, 2016, p.26). 


\section{Colorantes naturales}

Entre los colorantes naturales se encuentran: "los hidrosolubles, solubles en agua, los liposolubles o solubles en la grasa, y los minerales” (Sánchez, 2013, p.238).

Tabla 1

Clasificación de colorantes naturales

\begin{tabular}{|l|l|}
\hline \multicolumn{2}{|c|}{ Colorantes naturales hidrosolubles } \\
\hline Curcumina (E100) & Riboflavina, lactoflavina o B2 (E101) \\
\hline Cochinilla o ácido caminico (E120) & Caramelo (E150) \\
\hline Betanina o rojo remolacha (E162) & Antocianos (E163) \\
\hline \multicolumn{2}{|c|}{ Colorantes naturales liposolubles } \\
\hline Clorofilas (E140 y 141) & Carotenoides (E160) \\
\hline Xantofilas (E161) & \\
\hline \multicolumn{2}{|c|}{ Minerales } \\
\hline Carbón vegetal (E153) & Carbonato cálcico (E170) \\
\hline Dióxido de titanio (E171) & Óxidos e hidróxidos de hierro (E172) \\
\hline Aluminio (E173) & Plata (E174) \\
\hline Oro (E175) & \\
\hline
\end{tabular}

Fuente: Sánchez et ál. (2013).

\section{Colorante natural antociano}

Los colorantes naturales antociano constituyen:

El grupo más difundido de colorantes vegetales, se localizan principalmente en las flores y frutos de las plantas superiores, sus colores varían entre el rojo violeta y azul [...] son especies solubles en agua, cuyo color y estabilidad quedan afectados considerablemente por el $\mathrm{pH}$, con un $\mathrm{pH}$ cercano a 1 presenta un color brillante y una gran estabilidad a la luz y temperatura, a medida que el medio se hace más alcalino, con pH cercanos al 3.5, el color adquiere un tono azulado y menor estabilidad, al igual que su fuerza de tinción decae bruscamente, sobre pH 4 las antocianinas se degradan rápidamente, por lo que sus aplicaciones funcionan mejor en productos ácidos como jugos y bebidas. (Flavorix, 2012)

Las antocianinas más comunes se obtienen por extracción de productos como moras, fresas, grosellas, uvas, frambuesas o maíz negro, de los cuales se elaboran bebidas, queso, confituras, helados de agua, yogur, productos lácteos aromatizados, entre otros (Sanchez, 2013). Estos colorantes no general efectos secundarios al consumidor, por lo que son "una excelente alternativa para los colorantes artificiales carmoisina, rojo 40 (allura red AC) y ponceau 4R” (Flavorix, 2013). 
Igualmente, las antocianinas son "investigadas por su rol antioxidante, se les atribuyen efectos benéficos sobre algunas funciones metabólicas e incluso una relación directa con la prevención de ciertas enfermedades cardiaca” (Flavorix, 2012).

Por lo tanto, el objetivo de este artículo es verificar el poder de tinción del colorante natural antociánico en un yogur natural, comparándolo con un colorante artificial (rojo allura). El estudio analiza la obtención del colorante antocianico partiendo del residuo mora de castilla y se describen los equipos utilizados, condiciones de operación y método de extracción; asimismo, se realizan pruebas de estabilidad (temperatura, luz y $\mathrm{pH}$ ) con el fin de identificar las condiciones apropiadas para el almacenamiento del colorante natural.

\section{METODOLOGÍA}

\section{Obtención del colorante natural}

- Secar el residuo de la mora de castilla en una mufla a $40 \pm 0.1^{\circ} \mathrm{C}$ durante $48 \mathrm{~h}$.

- La extracción de antocianinas se lleva a cabo con el residuo mora de castilla, previamente seco y triturado, realizando una extracción soxhlet en la que se utiliza como disolvente etanol al 96\% (v/v) y etanol recuperado al 94\% (v/v).

- Para purificar el extracto se utiliza un rotaevaporador Heidolph, programado a una temperatura de $60{ }^{\circ} \mathrm{C}$ y a una presión de 175 mbar.

- Se realiza un ensayo ácido base, que permite verificar la presencia de antocianinas en el extracto.

- Para cuantificar las antocianinas se utiliza espectrofotometría Jenway 6320D, partiendo de soluciones buffer. Para la cuantificación de las antocianinas totales se empleó el método de $\mathrm{pH}$ diferencial; sus resultados se expresan en mg cianidin-3-glucosido sobre $100 \mathrm{~g}$ de residuo.

Una vez obtenido el colorante natural, proveniente de la extracción de antocianinas del residuo de mora de castilla, se realizan pruebas de estabilidad para seleccionar las mejores condiciones de conservación de este colorante.

\section{Pruebas de estabilidad}

Se realiza ensayos para probar la estabilidad del colorante, en los que se modifica la acidez y la basicidad del colorante; también se varía la temperatura a la que se almacenaba la muestra y la cantidad de luz a la que se expone durante el almacenamiento.

El colorante natural es sometido a diferentes condiciones de temperatura, $\mathrm{pH}$ y luz durante cuatro días; pasado este tiempo, se realiza la lectura de absorbancias a $540 \mathrm{~nm}$

y $700 \mathrm{~nm}$ de los extractos, utilizando las soluciones buffer de acetato de sodio y cloruro de potasio. Finalmente se realiza la cuantificación de antocianinas. 
En tres envases ámbar se adicionan $3 \mathrm{~mL}$ de muestra, que se someten a diferentes condiciones de $\mathrm{pH}$. Para el $\mathrm{pH}$ ácido se adiciona gotas de ácido clorhídrico concentrado (37\%) hasta obtener un $\mathrm{pH}$ de 2, y para el $\mathrm{pH}$ alcalino se prepara una solución de bicarbonato de sodio al 5\%y se adiciona gotas hasta $\mathrm{pH}$ de 5 .

\section{Temperatura}

En tres envases ámbar se adiciona $3 \mathrm{~mL}$ de muestra y cada uno se lleva a diferentes condiciones de temperatura: congelación $\left(-10 \pm 2^{\circ} \mathrm{C}\right)$, refrigeración $\left(4 \pm 2^{\circ} \mathrm{C}\right) \mathrm{y}$ ambiente $\left(16 \pm 3^{\circ} \mathrm{C}\right)$.

\section{LuZ}

Se adicionan $3 \mathrm{~mL}$ de colorante en 3 envases translúcidos y se mantiene a una temperatura ambiente de $16 \pm 3{ }^{\circ} \mathrm{C}$ : el primer envase se expone a luz fluorescente generada por un bombillo de $70 \mathrm{~W}$ y a una distancia de $10 \mathrm{~cm}$; la segunda muestra se almacena en una caja sin presencia de luz, y en el tercer envase se deja el extracto en luz ambiente.

\section{Estabilidad del colorante en el yogur}

Para verificar el poder de tinción que presentan las muestras obtenidas utilizando etanol como solvente, se adiciona $0.3 \mathrm{~mL}$ de extracto en $10 \mathrm{ml}$ de yogur de Pasco natural, según lo propuesto por Ramírez, Rojas y Correo (2006), y para homogenizar se realiza agitación magnética durante 2 min a 500 rpm. El desempeño del colorante se evalúa en el yogur natural, para observar cómo se comporta en un producto alimenticio con un $\mathrm{pH}$ poco favorable para el colorante, que en el yogur tiene un $\mathrm{pH}$ de 4.5, factor que afecta la estabilidad; esto quiere decir que, si el colorante natural presenta un desempeño favorable en el yogur, en productos alimenticios con $\mathrm{pH}$ inferior a 4.5 se presentará un mejor desempeño.

\section{Preparación del colorante artificial}

Se realiza la preparación del colorante artificial rojo allura (R 40), el cual es el más utilizado en la industria de alimentos para mejorar las propiedades organolépticas de jugos, yogures, mermeladas, entre otros. El colorante se encuentra en estado sólido, por esta razón es necesario preparar una disolución del artificial al 3\%, en la que se adicionan $0.3 \mathrm{~g}$ del colorante en $10 \mathrm{~mL}$ de agua destilada y se compara la tinción con el colorante natural; posteriormente, se realiza una lectura de absorbancia de la solución del colorante artificial y del colorante natural a $540 \mathrm{~nm}$ para verificar que no hay 
variación en la tinción de los colorantes. Se adicionan $0.3 \mathrm{~mL}$ de la solución colorante artificial en $10 \mathrm{~mL}$ de yogur.

La comparación del poder de tinción del colorante natural en el yogur con respecto al colorante sintético y bajo las mismas condiciones se realiza durante un mes, en el que cada dos días se efectúa la lectura de absorbancia para cada una de las muestras a una longitud de onda de $540 \mathrm{~nm}$ y se verifica si hay degradación de color. Para este proceso también se tiene en cuenta el colorante natural que se obtiene con etanol recuperado $94 \%(\mathrm{v} / \mathrm{v})$.

\section{RESULTADOS Y DISCUSIÓN}

\section{Obtención del colorante natural}

Antes de realizar el proceso de secado es necesario verificar el pH del mismo. Según lo reportado por Paes, Dotta y Martínez (2013), se debe garantizar un pH de 2.8, utilizando como agente acidificante solución de acuosa de ácido cítrico al 10\%.

\section{Figura 1}

Residuo de mora seco.

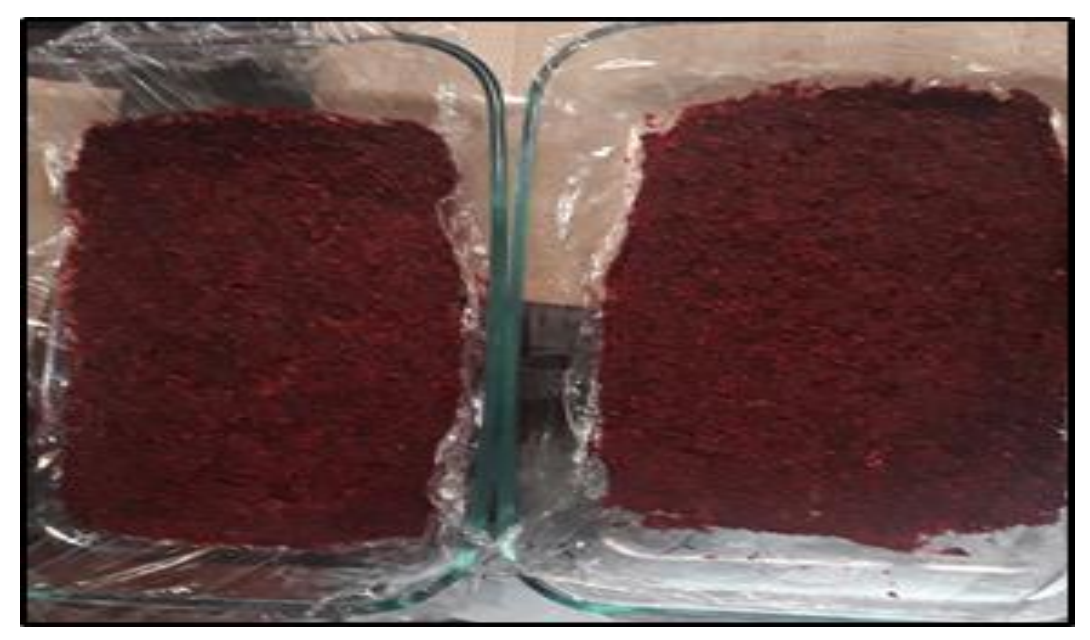

Para realizar la respectiva extracción de antocianinas, responsables de las tonalidades rojizas del colorante, se utiliza el método de extracción soxhlet y se usa como solvente etanol. En este proceso es necesario controlar la temperatura para evitar la ebullición violenta del solvente. El proceso de extracción se lleva a cabo durante 4 h. 
Figura 2

Extracción del colorante antocianico.

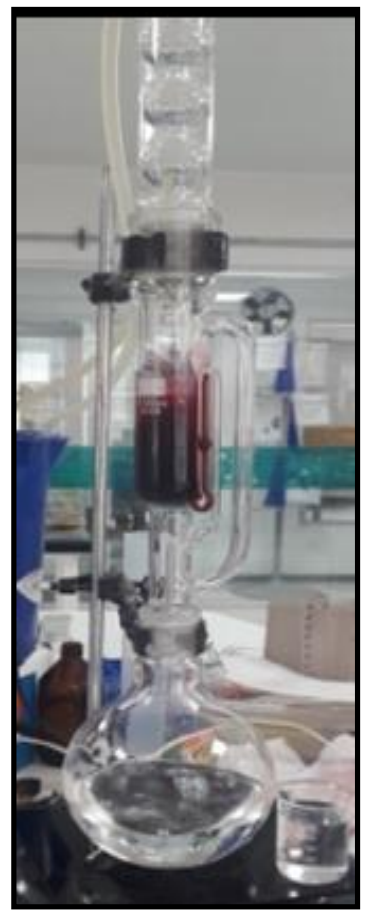

Figura 3

Purificación del colorante.

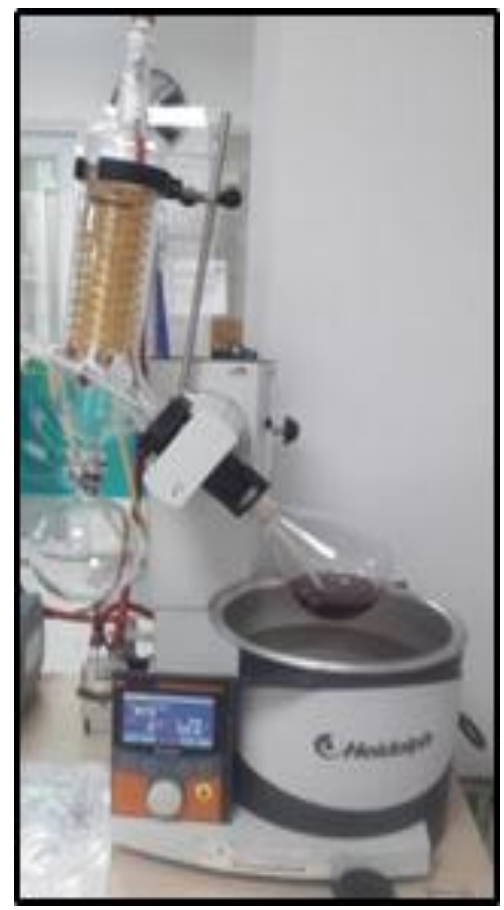

Por último, para la purificación del colorante natural se ingresa el extracto proveniente del método de extracción soxhlet al equipo Heidolph, el que se encarga de separar el colorante natural del solvente (etanol).

\section{Pruebas de estabilidad}

A continuación, se presentan las tablas con los resultados obtenidos bajo las diferentes condiciones de almacenamiento de las muestras. Se reporta el resultado para antocianinas monomericas y totales expresadas en mg cianidin-3-glucosido/100 g de residuo de mora.

\section{Tabla 2}

Resultado de las muestras sometidas a luz directa

\begin{tabular}{ccc}
\hline Antocianinas & $\begin{array}{c}\text { Soxhlet } \\
\text { E. 96\% }\end{array}$ & $\begin{array}{c}\text { Soxhlet } \\
\text { E.R }^{1}\end{array}$ \\
\hline Monoméricas & 57.611 & 50.097 \\
Totales & 131.086 & 110.213 \\
\hline
\end{tabular}

Nota. Contenido de antocianinas monomericas y totales de las muestras sometidas a luz directa. 
Bustos y Muñoz Betancourt

Tabla 3

Resultado de las muestras sometidas a oscuridad

\begin{tabular}{ccc}
\hline Antocianinas & $\begin{array}{c}\text { Soxhlet E. } \\
\mathbf{9 6 \%}\end{array}$ & Soxhlet E.R \\
\hline Monoméricas & 814.906 & 694.674 \\
Totales & 1004.438 & 772.323 \\
\hline
\end{tabular}

Tabla 4

Resultado de las muestras sometidas a luz ambiente

\begin{tabular}{ccc}
\hline Antocianinas & $\begin{array}{c}\text { Soxhlet } \\
\text { E. 96\% }\end{array}$ & $\begin{array}{c}\text { Soxhlet } \\
\text { E.R }\end{array}$ \\
\hline Monoméricas & 740.596 & 607.839 \\
Totales & 958.516 & 717.217 \\
\hline
\end{tabular}

En las tablas de resultados se evidencia que la luz directa sí afecta la estabilidad de las antocianinas, como se había mencionado en el marco teórico. De manera que en presencia de luz hay una degradación del compuesto de hasta un $87 \%$ en comparación con los resultados obtenidos en completa oscuridad y, por ende, se pierde intensidad en el color. Los mejores resultados se obtuvieron con las muestras en completa oscuridad; sin embargo, si se almacenan los extractos en envases translucidos a luz ambiente, se conserva la tinción del colorante, aunque hay una degradación de las antocianinas totales en un $5 \%$.

Tabla 5

Resultado de las muestras sometidas a refrigeración

\begin{tabular}{ccc}
\hline Antocianinas & $\begin{array}{c}\text { Soxhlet E. } \\
\mathbf{9 6 \%}\end{array}$ & Soxhlet E.R \\
\hline Monoméricas & 864.167 & 815.741 \\
Totales & 1062.884 & 940.982 \\
\hline
\end{tabular}

Tabla 6

Resultado de las muestras sometidas a congelación

\begin{tabular}{ccc}
\hline Antocianinas & $\begin{array}{c}\text { Soxhlet } \\
\text { E. 96\% }\end{array}$ & $\begin{array}{c}\text { Soxhlet } \\
\text { E.R }\end{array}$ \\
\hline Monoméricas & 526.015 & 449.200 \\
Totales & 623.703 & 533.529 \\
\hline
\end{tabular}


Estabilidad y desempeño del colorante antocianico en yogur natural

Tabla 7

Resultado de las muestras sometidas a temperatura ambiente

\begin{tabular}{ccc}
\hline Antocianinas & $\begin{array}{c}\text { Soxhlet } \\
\text { E. 96\% }\end{array}$ & $\begin{array}{c}\text { Soxhlet } \\
\text { E.R }\end{array}$ \\
\hline Monoméricas & 671.295 & 630.383 \\
Totales & 821.585 & 753.120 \\
\hline
\end{tabular}

Se analiza que la temperatura tiene un efecto negativo en la estabilidad de las antocianinas, que se ven mayormente afectadas a temperaturas de congelación, lo que ocasiona una degradación de las antocianinas totales de hasta un $41 \%$ en comparación con temperatura de refrigeración. Por su parte, a temperatura ambiente, las antocianinas se degradan en un $23 \%$; por esta razón, las muestras presentan un mejor comportamiento manteniendo los extractos a una temperatura de $4{ }^{\circ} \mathrm{C}$ y almacenadas en envases ámbar.

Tabla 8

Resultado de las muestras sometidas a $\mathrm{pH} 2$

\begin{tabular}{ccc}
\hline Antocianinas & $\begin{array}{c}\text { Soxhlet } \\
\text { E.96\% }\end{array}$ & $\begin{array}{c}\text { Soxhlet } \\
\text { E.R }\end{array}$ \\
\hline Monoméricas & 509.316 & 354.016 \\
Totales & 648.752 & 529.355 \\
\hline
\end{tabular}

Tabla 9

Resultado de las muestras sometidas a pH 5

\begin{tabular}{ccc}
\hline Antocianinas & $\begin{array}{c}\text { Soxhlet } \\
\text { E. 96\% }\end{array}$ & $\begin{array}{c}\text { Soxhlet } \\
\text { E.R }\end{array}$ \\
\hline Monoméricas & 240.464 & 64.291 \\
Totales & 298.075 & 109.378 \\
\hline
\end{tabular}

Sobre el efecto del pH en la estabilidad de las antocianinas, se logró comprobar que con $\mathrm{pH}$ ácido se conservan las antocianinas; por el contrario, a pH 5 hay degradación del compuesto de hasta un 54\% y el color del extracto cambia. Los resultados indican que la mayor retención de color se obtiene cuando el colorante está a un pH ácido, en refrigeración y en total oscuridad; sin embargo, al incorporar el colorante natural en un alimento no es posible mantener esta condición; por esto se recomienda almacenar a la luz ambiente, en la que también se presentan resultados favorables.

\section{Estabilidad del colorante en el yogur}

En la Figura 5 se evidencia que hay degradación del colorante natural; sin embargo, se observa que después de la primera semana las absorbancias se mantienen más constantes. Por su parte, cuando se examinan las Figuras 4 y 5 se observa una pérdida 
de tonalidad poco visible. Por otro lado, la tonalidad de la muestra nombrada como colorante natural S. 96\% es similar a la muestra soxhlet E. recuperado; esta comparación se realiza para garantizar que la reutilización del solvente recuperado no afectará el poder de tinción del producto final.

\section{Figura 4}

Estabilidad del colorante en el yogur

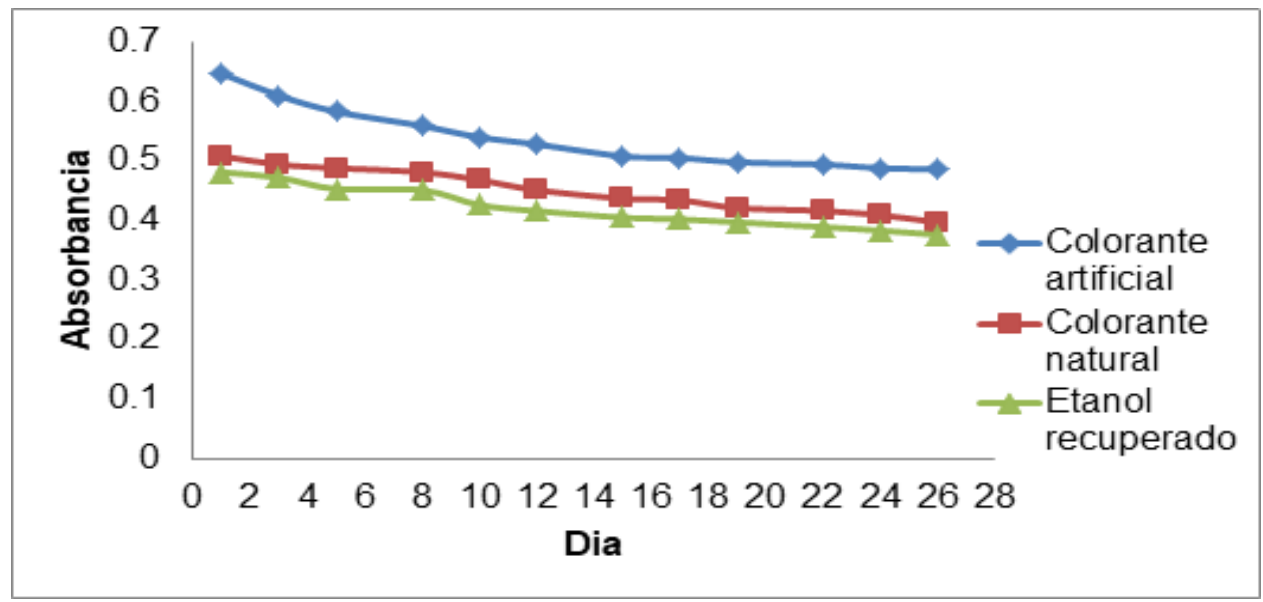

$\mathrm{Al}$ analizar la Figura 4 se puede observar que el colorante natural pierde poder de tinción en el yogur en hasta un $22 \%$ después de analizar la muestra durante un mes. Este porcentaje de perdida de tinción también es generado por el colorante natural obtenido con etanol recuperado al 94\%. De esta forma se garantiza que al recuperar el etanol y utilizar nuevamente en el proceso de extracción no se presentarán cambios en la calidad del colorante natural.

\section{Figura 5}

Estabilidad de las muestras día uno

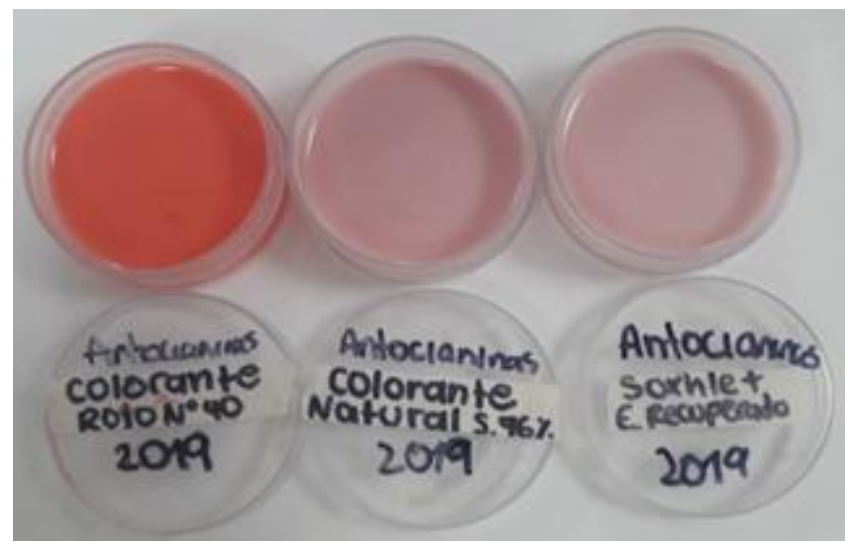




\section{Figura 6}

Estabilidad de las muestras día veinte seis

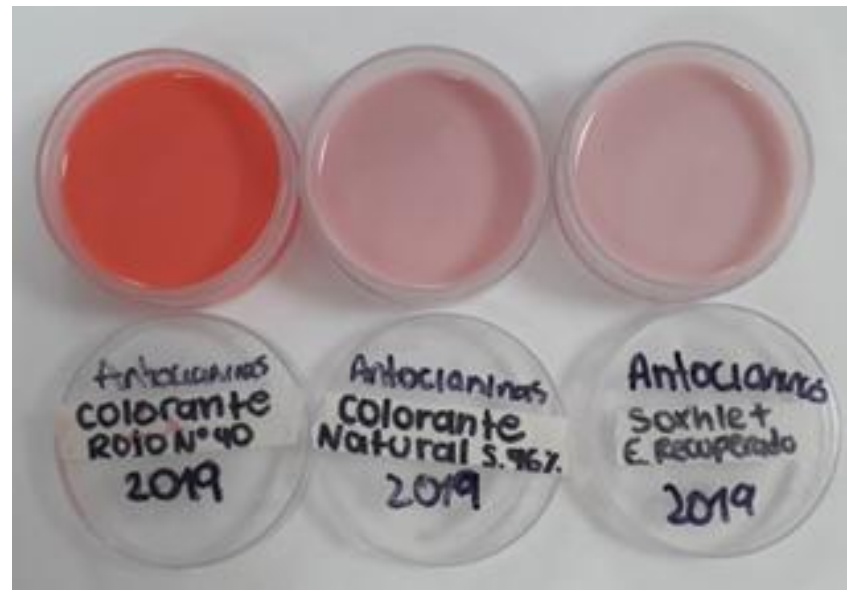

La degradación del colorante incorporado en el yogur se debe al pH del mismo, que es aproximadamente de 4.3, mientras que el $\mathrm{pH}$ del colorante natural es de 2.8. De manera que, como ya se había mencionado anteriormente, uno de los factores que afecta la estabilidad de las antocianinas es el pH. Esta información se comprobó también con las pruebas de estabilidad que se realizaron a pH 5, datos reportados en la tabla 9.

\section{CONCLUSIONES}

El colorante natural se debe almacenar en envases ámbar a una temperatura de $4{ }^{\circ} \mathrm{C}$ y $\mathrm{pH}$ inferior a 3 y no tiene que ser expuesto a luz directa. Esto con el fin de garantizar la calidad del colorante, lo que evita la degradación de las antocianinas, que es el componente principal que genera el color característico.

Se logró identificar la pigmentación del colorante en un producto alimenticio, en este caso, yogur natural, pasado un mes en análisis de estabilidad del color. Hay degradación del colorante que se verifica por espectrofotometría; esto se debe al pH del yogur de 4.5, factor que no favorece la estabilidad del colorante natural.

El colorante artificial presenta mayor poder de tinción que el natural; sin embargo, el colorante natural tiene mayores beneficios para la salud y no tiene restricciones de consumo, como sí los presenta el artificial.

\section{Referencias}

Belmonte, J. Arroyo, I., Vázquez, M., Cruz, E., y Peña, E. (2016). Colorantes artificiales en alimentos. Revista Naturaleza y Tecnología, 10, 24-38. http://quimica.ugto.mx/index.php/nyt/article/viewFile/204/pdf 
Elmadfa, I., Muskat, E., y Fritzsche, D. (2011). Tabla de aditivos. Los números. Hispano Europea S. A. Editorial.

Flavorix. (2012). Colorante Natural Antocianina (E-163). http://flavorix.com/producto/colorante-natural-antocianina-e-163/

Garzón, G. (2008). Las antocianinas como colorantes naturales y compuestos bioactivos: revisión alimentaria. Acta Biológica Colombiana, 13(3), 27-36.

Lucero, A., y Muñoz, A. (2019). Evaluación de la viabilidad técnico-financiera para la obtención de un colorante natural partiendo del residuo mora de castilla (Rubus glaucus Benth) para la aplicación en la industria de alimentos (tesis de pregrado). Programa de Ingeniería Química, Facultad de Ingenierías, Fundación Universidad de América, Bogotá D. C., Colombia.

Parra, V. (2004). Estudio comparativo de un colorante natural y sintético en alimentos desde el punto de vista funcional y toxicológico (tesis de pregrado). Escuela de Ingeniería de Alimentos, Facultad de Ciencias Agrarias, Universidad austral de Chile.

Ramírez, M., Rojas, N., y Correa, L. (2006) Obtención de un colorante natural alimentario de mora de castilla. Ciencia y Desarrollo, 2(2), p.115-130.

Sánchez, J. (2013). La química del color en los alimentos. Química Viva, 12(3), 234246. https://www.redalyc.org/pdf/863/86329278005.pdf

Sanz, A. (s. f.). La industria de los colorantes y pigmentos. https://www.eii.uva.es/organica/qoi/tema-11.php

Paes, J., Dotta, R., y Martínez, J. (2013). Extraction of phenolic compounds from blueberry (Vaccinium myrtillus L.) residues using supercritical $\mathrm{CO}_{2}$ and pressurized water. En III Iberoamerican Conference on Supercritical Fluids. Conferencia realizada en Cartagena de Indias, Colombia. 